\title{
Considerações sobre a relação entre a hipertensão e o prognóstico da COVID-19
}

\section{Considerations about the relationship between hypertension and the prognosis of COVID-19}

\author{
Gabriel Martins de $\operatorname{Barros}^{1}$ (D) João Batista Raposo Mazullo Filho ${ }^{2,3}$ (D), Aírton Conde Mendes Júnior ${ }^{4}$ (D) \\ 1. Mestrando em Ciências e Saúde pela Universidade Federal do Piauí (UFPI), Teresina, PI, Brasil. 2. Docente do Centro Universitário Santo Agostinho \\ (UNIFSA), Teresina, PI, Brasil. 3. Especialista em Fisioterapia Cardiorrespiratória pela Universidade Tuiuti do Paraná (UTP), Curitiba, PR, Brasil. 4. Docente \\ do Departamento de Morfologia da Universidade Federal do Piauí (UFPI), Teresina, PI, Brasil
}

\section{Resumo}

Objetivo: iinvestigar a potencial associação entre a hipertensão arterial sistêmica (HAS) e a gravidade da COVID-19. Métodos: realizou-se uma pesquisa eletrônica no Medline, Scopus e Web of Science acerca da relação entre HAS e COVID-19. Resultados: as primeiras hipóteses levantadas revelaram que a associação entre as doenças estava no tratamento com inibidores da enzima de conversão da angiotensina e bloqueadores dos receptores da angiotensina. Contudo, em um estudo experimental, observou-se que os pacientes hipertensos tratados com esses medicamentos apresentaram menor taxa de gravidade da doença. Conclusão: até o momento, a relação de HAS e COVID-19 é conflitante.

Palavras-chave: Hipertensão. Coronavírus. Prognóstico.

\begin{abstract}
Objective: to investigate the potential association between systemic arterial hypertension (SAH) and the severity of COVID-19. Methods: An electronic research on Medline, Scopus and Web of Science was conducted about the relationship between SAH and COVID-19. Results: The first hypotheses raised revealed that the association between the diseases was in the treatment with angiotensin conversion enzyme inhibitors and angiotensin receptor blockers. However, in an experimental study it was observed that hypertensive patients treated with these drugs had a lower rate of disease severity. Conclusion: So far, the relationship between SAH and COVID-19 is conflicting.
\end{abstract}

Keywords: Hypertension. Coronavirus. Prognosis.

O novo coronavírus (COVID-19 ou SARS-CoV2) espalhou-se, rapidamente, pelo mundo, logo após sua primeira ocorrência em Wuhan, na China, em dezembro de $2019^{1}$. O SARS-CoV-2 pertence ao gênero $\beta$-coronavírus da família Coronaviridae e possui $96 \%$ de identidade genômica com um vírus de morcego semelhante ao Síndrome Respiratória Aguda Grave (SARS) detectado anteriormente ${ }^{2}$.

É fatal em $2 \%$ dos casos relatados ${ }^{3}$, mas porcentagens mais altas estão sendo estimadas em outros países, como a Itália. As diferenças entre os países se dão, provavelmente, devido a mudanças virais, número de testes feitos, tipo de sistemas de saúde, ações tomadas para conter o surto, e, também, diferenças na prevalência e nas características de condições que possam interagir com COVID-19 para piorar o prognóstico, como a idade da população e as comorbidades crônicas. 0 conhecimento do comportamento do vírus, dos fatores de risco que favorecem o desenvolvimento e a progressão do COVID-19 é crucial para analisar, precocemente, o que pode acontecer em todo o mundo no futuro ${ }^{4}$.

Entre os potenciais fatores de risco verificados até o momento está a hipertensão arterial sistêmica (HAS), uma das doenças crônicas mais comuns em humanos, que afeta mais de 1 bilhão de pessoas em todo o mundo, o que resulta em diversas complicações com consequências significativas para a saúde pública. Na população em geral, a prevalência de HAS é de cerca de 30 a 45\%, mas aumenta rapidamente com a idade avançada ${ }^{5}$. A influência da HAS em diversas patologias com impactos no sistema respiratório ainda não é bem compreendida, como é o caso da recente COVID-19.

O aumento da mortalidade e morbidade do COVID-19 em pacientes com HAS é uma associação que foi observada em estudos epidemiológicos realizados até o momento. Dados extraídos do Sistema de Informação de Doenças Infecciosas da China, relatados até 11 de fevereiro de 2020, com um total de 72.314 prontuários, mostram que a maioria das mortes e complicações são em pacientes com 60 anos ou mais que sofrem de doenças subjacentes, como hipertensão, doenças cardiovasculares e diabetes ${ }^{6}$. Em outro estudo de coorte retrospectivo de 201 pacientes com pneumonia confirmada por COVID-19, 84 deles desenvolveram síndrome do desconforto respiratório agudo, e destes 23 apresentavam a HAS como comorbidade, sendo a condição crônica mais comum nessa amostra?.

Na pandemia de 2003 com SARS, relatórios mostraram que alguns fatores não genéticos, como condições mórbidas (incluindo HAS), representam fatores de risco para o desenvolvimento da insuficiência respiratória com SARS ${ }^{8}$. É importante evidenciar que, geralmente, os indivíduos que apresentam HAS são idosos 
e sedentários, fatores que podem ocasionar uma confusão de dados, implicando que a HAS propriamente dita não está correlacionada com a COVID-19.

Pesquisas atuais apontam que a relação da HAS com o novo coronavírus ocorre por seu tratamento com medicamentos antihipertensivos específicos: inibidores da enzima de conversão da angiotensina (IECAs) e bloqueadores dos receptores da angiotensina (BRA) ${ }^{9}$. A enzima conversora de angiotensina 2 (ACE2) é a porta de acesso para o SARS-CoV-2 entrar nas células humanas4. E, no sistema renina-angiotensina, é desempenhado um papel fundamental na manutenção da homeostase da pressão arterial, bem como no balanço hídrico e salino em mamíferos, e a ativação anormal desse sistema tem sido associada a diversas patogêneses ${ }^{10}$.

As quantidades circulantes de ACE2 são aumentadas em pacientes com hipertensão, e os níveis são aumentados ainda mais por diferentes drogas, incluindo IECAs e BRA9. Relatórios preliminares mostraram que pacientes com COVID-19 aumentaram a angiotensina II em comparação com pessoas saudáveis. O aumento anormal da angiotensina II foi relacionado à hipertensão e à insuficiência pulmonar ${ }^{11,12}$.

Também deve ser enfatizado que alelos específicos controlam a expressão, a atividade e a resposta da ECA2 aos IECAs, pois pesquisas em modelos animais e seres humanos que receberam IECA, BRA, ou ambos aumentaram os níveis de ACE2 nas células luminais do intestino9,13. Nessa perspectiva, deve-se notar que a modificação da glicosilação é essencial para a ligação da proteína spike SARS-CoV-2. Em decorrência dessa temática, foi iniciado, por meio de investigações e até inclusão experimental por governo de diversos países, o uso de cloroquina, hidrocloroquina ou outro inibidor de serinoproteases que possa reduzir, significativamente, a infecção das células hospedeiras por SARS-CoV-2 in vitro ou pacientes, visando à inibição da glicosilação de ACE2 ${ }^{9,13}$.

Entretanto, no estudo de Meng et al. ${ }^{12}, 42$ pacientes foram divididosem doisgruposcombaseemterapias anti-hipertensivas: um grupo de pacientes tratados com medicamentos IECA ou BRA, e outro grupo que incluiu pacientes tratados com outros anti-hipertensivos medicamentos, incluindo bloqueadores dos canais de cálcio, betabloqueadores e diuréticos. Foi observado que os pacientes que receberam terapia com IECA ou BRA apresentaram menor taxa de doenças graves e tendência a menor nível de IL-6 no sangue periférico. Além disso, a terapia com IECA ou BRA aumentou as contagens de células T CD3 e CD8 no sangue periférico e diminuiu o pico de carga viral em comparação a outro grupo. Nesse estudo, permanece incerto se há associação das patologias.

Fisiologicamente, a HAS tende a causar uma redução da capacidade respiratória. A tensão desenvolvida na parede ventricular esquerda durante a ejeção é anormalmente elevada; o concêntrico resultante da hipertrofia ventricular esquerda leva a uma redução no volume e na complacência da câmara e, por consequência, um aumento na pressão diastólica final do ventrículo esquerdo. Isso torna o preenchimento ventricular mais difícil e, assim, causa uma contração atrial rápida. 0 resultado é uma distensão transitória das veias pulmonares e aumento da pressão capilar pulmonar. Acima de um valor crítico, pode levar a vazamento de soro no espaço intersticial pulmonar. Além disso, existe um retrocesso efeito sobre a pressão arterial pulmonar, que é ligeiramente superior ao normal. Dessa forma, a HAS apresenta dois fatores principais que podem afetar a ventilação pulmonar: distensão vascular pulmonar e aumento do líquido intersticial pulmonar, que levam à resistência das pequenas vias aéreas e à influência no calibre bronquiolar, diminuindo, assim, a capacidade respiratória ${ }^{14}$.

Uma pesquisa investigou 38 mulheres e 11 homens com média de idade de $60 \pm 9$ anos e presença de HAS. A análise da respiratória dos pacientes ocorreu por meio da espirometria, PeakFlow, Força Muscular Respiratória e o Teste de caminhada de 6 minutos. Observou-se que os indivíduos apresentaram diminuição significativa no pico de fluxo expiratório, na força muscular respiratória e na tolerância ao exercício ${ }^{15}$. Contudo, deve-se levar em consideração que os resultados deste estudo são limitados pela inexistência de um grupo controle e idade avançada dos indivíduos.

Conforme os primeiros dados, a literatura aponta para resultados controversos da associação de medicamentos de IECAs e BRA para tratamento de HAS e prognóstico do COVID-19. É verificada, em alguns estudos, uma redução da capacidade ventilatória em indivíduos com HAS, o que pode agravar a saúde de um indivíduo acometido; além disso, a associação da doença com outras morbidades respiratórias, parece demonstrar a afinidade da HAS e o sistema respiratório. Vale ressaltar, também, que a HAS foi comum na morbimortalidade da pandemia de SARS em 2003, mesmo que sua associação ainda não seja entendida. A situação está evoluindo progressivamente; o acompanhamento rigoroso de informações é importante para a comunicação correta com pacientes e profissionais, sendo necessário delinear melhor o sistema renina-angiotensina, principalmente a ACE2 na patogênese do COVID-19.

Pesquisas futuras devem mostrar um olhar voltado para a capacidade respiratória funcional de indivíduos com HAS, que parece estar diminuída; inclusive, estudos voltam-se para a adoção de planos de tratamentos, visando à reabilitação respiratória e não somente à cardíaca. É primordial que indivíduos que possuem HAS continuem com sua medicação neste momento de pandemia, além da prática regular de exercícios físicos (domiciliar) com possível orientação médica ou fisioterapêutica; que façam manutenção da hidratação; tenham alimentação saudável; sono moderado e controle do estresse.

\section{REFERÊNCIAS}

1. Wong J, Goh QY, Tan Z, Lie SA, Tay YC, Ng S Y, et al. Preparing for a COVID-19 pandemic: a review of operating room outbreak response measures in a large tertiary hospital in Singapore. Can J Anesth/J Can Anesth. 2020 Mar. 1-14. doi:10.1007/s12630-020-01620-9. 


\section{Hipertensão e COVID-19}

2. Zehender G, Lai A, Bergna A, Meroni L, Riva A, Balotta C, et al. Genomic characterisation and phylogenetic analysis of Sars-Cov-2 in Italy. J Med Virol. 2020 Mar. doi:10.1002/jmv.25794.

3. Mahase E. Coronavirus: covid-19 has killed more people than SARS and MERS combined, despite lower case fatality rate. BMJ; 2020 Fev; 368: m641. doi:10.1136/bmj.m641.

4. Maddaloni E, Buzzetti R. Covid-19 and diabetes mellitus: unveiling the interaction of two pandemics. Diabetes Metab Res Rev. 2020 Mar: e33213321. doi:10.1002/dmrr.3321.

5. Kallikazaros I E. Arterial hypertension. Hellenic J Cardiol. 2013 Set-Out; 54(5): 413-416.

6. Zhonghua Liu Xing Bing XueZaZhi. [The epidemiological characteristics of an outbreak of 2019 novel coronavirus diseases (COVID-19) in China]. Novel Coronavirus Pneumonia Emergency Response Epidemiology Team. 2020 Fev; 41(2): 145-51. doi:10.3760/cma.j.issn.0254-6450.2020.02.003.

7. Wu C, Chen X, Cai Y, Zhou X, Xu S, Huang H, et al. Risk factors associated with acute respiratory distress syndrome and death in patients with coronavirus disease 2019 pneumonia in Wuhan, China. JAMA Intern Med. 2020 Mar. doi:10.1001 / jamainternmed.2020.0994.

8. Zhi L, Zhou G, Zhang H, ZhaiY, Yang H, Zhang F, et al. Lack of support for an association between CLEC4M homozygosity and protection against SARS coronavirus infection. Nat Genet. 2007 Jun; 39(6): 692-693. doi: 10.1038/ ng0607-691.
9. Fang L, Karakiulakis G, Roth. Antihypertensive drugs and risk of COVID-19?Authors' reply. Lancet. 2020 Mar. doi:10.1016/S2213-2600.

10. KubaK, Imai Y, Ohto-NakanishiT, Penninger J M. Trilogy of ACE2: A peptidase in the renin-angiotensin system, a SARS receptor, and a partner for amino acid transporters. Pharmacol Ther. 2010 Out; 128(1): 119-128. doi:10.1016 / j.pharmthera.2010.06.003.

11. Liu Y, Yang Y, Zhang C, HuangF, Wang F, Yuan J, et al. Clinical and biochemical indexes from 2019-nCoV infected patients linked to viral loads and lung injury. Sci China Life Sci. 2020 Mar; 63(3): 364-374.doi:10.1007 / s11427-020-1643-8.

12. Meng J, Xiao G, Zhang J, He X, Ou M, Bi J, et al. Renin-angiotensin system inhibitors improve the clinical outcomes of COVID-19 patients with hypertension. Emerg Microbes Infect. 2020 Dez; 9(1): 757-760. doi:10.1080 / 22221751.2020.1746200.

13. Vuille-dit-Bille RN, Camargo SM, Emmenegger L, Sasse T, Kummer E, Jando $J$, et al. Human intestine luminal ACE2 and amino acid transporter expression increased by ACE-inhibitors. Amino Acids. 2015 Abr; 47(4): 693-705. doi:10.1007 / s00726-014-1889-6.

14. Bucca C, Rolla G, Pecchio O, Ratti C, Accatino G, Galeasso, B. Systemic Arterial Hypertension and Small Airways Function. Respiration 1980; 39(4): 188192. doi:10.1159/000194222.

15. Ruas G, Couto VF, Pegorari MS, Ohara DG, Jamami LK, Jamami M. Avaliação respiratória, capacidade funcional e comorbidade em indivíduos com hipertensão arterial. Rev. Saúde Coletiva 2013; 10(59): 31-36. 